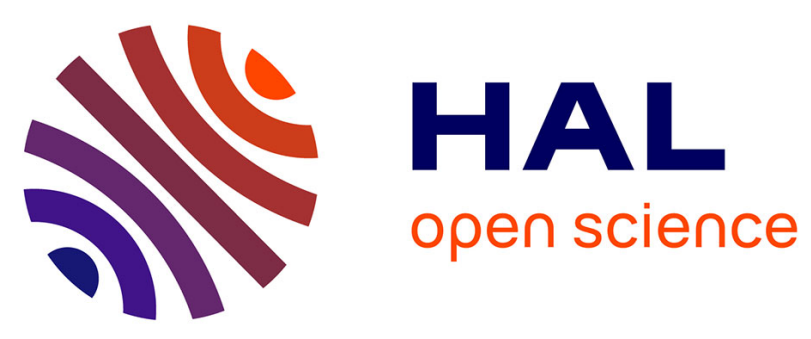

\title{
Sur le contact métal-isolant
}

Roland Coelho, Nasreddine Bouguila

\section{To cite this version:}

Roland Coelho, Nasreddine Bouguila. Sur le contact métal-isolant. Revue de Physique Appliquée, 1988, 23 (9), pp.1467-1474. 10.1051/rphysap:019880023090146700 . jpa-00245972

\section{HAL Id: jpa-00245972 https://hal.science/jpa-00245972}

Submitted on 1 Jan 1988

HAL is a multi-disciplinary open access archive for the deposit and dissemination of scientific research documents, whether they are published or not. The documents may come from teaching and research institutions in France or abroad, or from public or private research centers.
L'archive ouverte pluridisciplinaire HAL, est destinée au dépôt et à la diffusion de documents scientifiques de niveau recherche, publiés ou non, émanant des établissements d'enseignement et de recherche français ou étrangers, des laboratoires publics ou privés. 
Classification

Physics Abstracts

$68.48-73.40 \mathrm{~N}-77.30$

\title{
Sur le contact métal-isolant
}

\author{
Roland Coelho et Nasreddine Bouguila \\ Laboratoire de Physique des Décharges, ER 114 du C.N.R.S., Ecole Supérieure d’Electricité, 91190 Gif sur \\ Yvette, France
}

(Reçu le 15 octobre 1987, accepté le 19 juin 1988)

\begin{abstract}
Résumé. - Le présent article traite de quelques notions simples sur l'ouverture et la fermeture d'un contact entre un isolant et une pièce métallique portée à une haute tension, et précise l'importance des charges libres à l'interface. Un modèle électrostatique élémentaire d'un plan de charges mobiles, étendu dans deux annexes à une charge répartie dans l'épaisseur, permet de calculer l'évolution du potentiel de surface après l'ouverture $\mathrm{du}$ contact (déclin naturel) et après un court-circuit temporaire (retour du potentiel). L'interprétation des résultats à l'aide de ce modèle permet de caractériser l'injection de charges à l'interface, et d'estimer de façon simple la quantité et la position du centroïde des charges injectées, ainsi que la mobilité effective de ces charges.
\end{abstract}

\begin{abstract}
This work deals with simple concepts on the opening and closure of a contact between an insulator and metal surface stressed at high potential, and it emphazises the importance of the free charge at the interface. An elementary electrostatic model of a mobile charge plane, extended in two appendices to a distributed charge, enables to calculate the surface potential after the opening of the contact (natural decay) and after a temporary short circuit (return potential). Interpretation of experimental data by means of this model helps to characterize the charge injection at the interface, to evaluate simply the amount of injected charge, and to estimate the effective charge mobility.
\end{abstract}

\section{Introduction et expérimentation.}

L'objet du présent article est de préciser quelques notions élémentaires sur des transferts de charge entre un conducteur chargé sous haute tension et un isolant, ainsi que sur la dynamique des charges transférées après l'ouverture du contact.

Ces concepts ne sont qu'indirectement liés aux accélérateurs de particules, mais ils sont à la base du fonctionnement des générateurs électrostatiques qui tiennent lieu d'injecteurs, et l'expérience montre que les notions en question sont parfois assez mal assimilées. Ainsi, nous n'avons pas hésité à simplifier au maximum les configurations étudiées, pour essayer de dégager les points essentiels, et en particulier le fait que les lois classiques de l'électrostatique ne permettent pas seulement de poser des problèmes aux étudiants, mais également d'interpréter des phénomènes qui semblent échapper à toute analyse quantitative.

Ainsi, le comportement d'une courroie isolante en contact périodique avec un rouleau conducteur porté à haute tension a été simulé dans ce travail par celui d'un film isolant sur un substrat à la masse, sur lequel une électrode métallique sous haute tension peut venir s'appuyer transitoirement.

Nous ne reviendrons pas sur les transferts de charge entre un métal et un isolant, qui ont été traités dans un grand nombre de publications [1-3], mais nous insisterons sur quelques expérimentations simples telles que :

a) l'étude de la charge acquise par un film isolant (polyéthylène, polyester, etc...) métallisé ou non, lors du contact avec une électrode de laiton portée à une tension $V$ (jusqu'à $6 \mathrm{kV}$ ) pendant des durées variables (de quelques secondes à quelques minutes) ;

b) l'étude de l'évolution du potentiel de surface du film isolant après ouverture du contact correspondant à la levée de l'électrode métallique sous tension ;

c) éventuellement, l'étude de l'évolution du potentiel de surface de l'échantillon après un courtcircuit temporaire de celui-ci. 
Le dispositif expérimental est schématisé sur la figure 1.

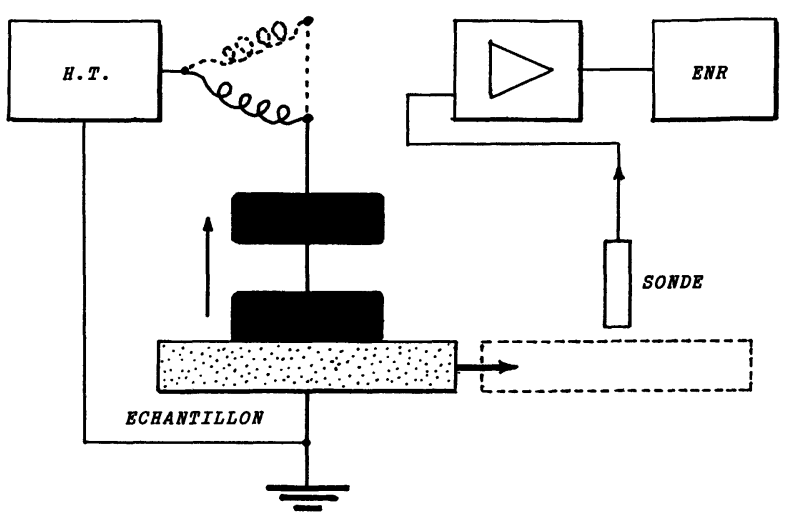

Fig. 1. - Schéma de l'ouverture d'un contact métal/isolant, avec la sonde de mesure du potentiel.

[Opening of the metal/insulator contact, with the potential probe.]

Le potentiel superficiel des échantillons métallisés peut être mesuré en reliant la métallisation à l'entrée d'un électromètre CARY 401 muni de son diviseur capacitif. Toutefois, pour uniformiser la technique de mesure, on a généralement utilisé un porteéchantillon coulissant, solidaire d'un vérin pneumatique, et permettant d'amener rapidement (en moins de $0,2 \mathrm{~s}$ ) l'endroit de l'échantillon qui était en contact avec l'électrode avant la remontée de celleci, sous la fenêtre d'une sonde asservie MONROE 1017. Ainsi, le potentiel de surface $v$ de l'échantillon peut être enregistré après un délai inférieur à $0,3 \mathrm{~s}$ après l'ouverture du contact.

\section{Modèle élémentaire de l'ouverture du contact.}

L'équipartition de la charge dans les condensateurs en série (échantillon et lame d'air), valable en l'absence de charges « libres » à l'interface, s'écrit :

$$
C v=C_{\mathrm{a}}(V-v)
$$

où $C=\varepsilon S / e$ est la capacité fixe de l'échantillon et $C_{\mathrm{a}}=\varepsilon_{0} S / y$ la capacité variable de la lame d'air. On négligera les effets de bord, ce qui réduira le problème à une dimension.

De (1), on tire :

$$
v=\frac{1}{1+C / C_{\mathrm{a}}} V
$$

où $C / C_{\mathrm{a}}=\kappa y / e\left(\kappa=\varepsilon / \varepsilon_{0}=\right.$ permittivité relative de l'échantillon).

2.1 CAS GÉNÉRAL D'UNE SÉPARATION A VITESSE QUELCONQUE. - Soit $y(t)$, avec $y(0)=0$, l'épaisseur de la lame d'air à l'instant $t$.
La relation (2) devient :

$$
v(t)=\frac{V}{1+\alpha y},
$$

avec

$$
\alpha=\kappa / e .
$$

Le champ dans l'échantillon est :

$$
E_{\mathrm{e}}(t)=\frac{V}{e(1+\alpha y)}=\frac{E_{\mathrm{e}}(0)}{1+\alpha y},
$$

et le champ dans la lame d'air est :

$$
E_{\mathrm{a}}(t)=\frac{V-v}{y}=\frac{\alpha V}{1+\alpha y}=\frac{E_{\mathrm{a}}(0)}{1+\alpha y} .
$$

Ainsi, dans la mesure où il n'y a pas de charges « libres » sur la surface, le champ dans la lame d'air excède à tout instant le champ dans l'échantillon par le facteur $\kappa=\varepsilon / \varepsilon_{0}$ :

$$
E_{\mathrm{a}}=\kappa E_{\mathrm{e}} .
$$

Ceci résulte de la continuité de l'induction à l'interface, et aurait d'ailleurs pu servir de point de départ au calcul.

On voit que la lame d'air est soumise à un champ $\kappa E_{\text {ech }}$ qui, si $E_{\text {ech }}$ est de l'ordre de $10^{4} \mathrm{~V} \mathrm{~m}^{-1}$, risque de faire "claquer » la lame d'air. Par exemple, avec $V=2 \mathrm{kV}, \kappa=3$ et $e=5 \times 10^{-5} \mathrm{~m} \quad(=50 \mu \mathrm{m})$, $\alpha=6 \times 10^{4} \mathrm{~m}^{-1}$, et $E(0)=1,2 \times 10^{8} \mathrm{~V} \mathrm{~m}^{-1}$, champ 50 fois supérieur à la rigidité diélectrique de l'air. Toutefois ce champ décroît rapidement comme $(1+\alpha t)^{-1}$ et devient de l'ordre de la rigidité de l'air en $1 \mathrm{~ms}$ environ.

\subsection{CAlCul Des Courants DE DÉPlacement. -} Nous allons maintenant calculer l'expression des courants de déplacement dans l'échantillon et la lame d'air.

Dans l'échantillon, le courant de déplacement est :

$$
i_{\mathrm{e}}=C \frac{\mathrm{d} v}{\mathrm{~d} t}=-\frac{\alpha C y^{\prime}}{(1+\alpha y)^{2}} V .
$$

D'autre part, en utilisant l'expression (3) pour $v(t)$ et en tenant compte du fait que $\mathrm{d} C_{\mathrm{a}} / \mathrm{d} t=$ - $\left(y^{\prime} / y\right) C_{\mathrm{a}}$, le courant de déplacement dans la lame d'air prend la même forme :

$$
i_{\mathrm{a}}=-\frac{\alpha C y^{\prime}}{(1+\alpha y)^{2}} V=i_{\mathrm{e}} .
$$

On retrouve le fait, général, que l'équipartition de la charge entre les condensateurs en série équivaut à la continuité du courant de déplacement. Cette équivalence n'est plus obéie s'il y a des charges libres (par exemple déposées par effet couronne) à la surface de l'échantillon. 
Il faut noter également que, vu son signe, le courant de déplacement n'est pas fourni mais absorbé par l'alimentation. Sa valeur initiale est $i(0)=-\alpha C y^{\prime} V$. Si le mouvement n'est pas progressivement accéléré mais, par exemple, à vitesse constante de l'ordre de $0,1 \mathrm{~m} / \mathrm{s}$, on peut estimer la grandeur initiale $i(0)$ du courant de déplacement en utilisant les valeurs numériques de la section 2.1, avec une surface de contact de l'ordre de $1 \mathrm{~cm}^{2}$ et pour $V=2 \mathrm{kV}$, à environ $7 \times 10^{-4} \mathrm{~A}$.

\subsection{INFLUENCE D'UNE COUCHE DE CHARGES LIBRES} À L'INTERFACE. - S'il existe une couche de densité $\sigma$ de charges « libres » à la surface de l'échantillon, la relation entre les champs $E_{\mathrm{a}}$ dans l'air et $E_{\mathrm{e}}$ dans l'échantillon, exprimant la continuité de l'induction doit être corrigée en considérant un petit cylindre de Gauss (Fig. 2) dont les faces sont situées de part et d'autre de l'interface. Il résulte de l'observation de ce cylindre de Gauss que :

$$
\varepsilon E_{\mathrm{e}}=\varepsilon_{0} E_{\mathrm{a}}+\sigma .
$$

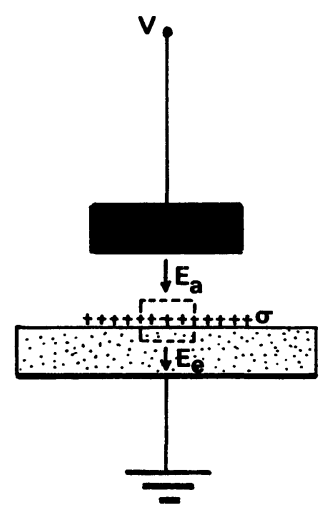

Fig. 2. - Champs électriques dans l'isolant et la lame d'air.

[Electric fields in the insulator and the air gap.]

Comme nous le verrons lors de la description des résultats expérimentaux, plusieurs observations militent effectivement en faveur de la présence d'une couche de charges libres à l'interface.

Ces observations, essentiellement au nombre de quatre, sont décrites à la section suivante.

\section{Observations expérimentales.}

Les observations mentionnées plus haut ont été les suivantes :

3.1 Charge maximale par contact. - La figure 3 représente le potentiel initial mesuré sur un échantillon de PET métallisé de $36 \mu \mathrm{m}$ d'épaisseur,

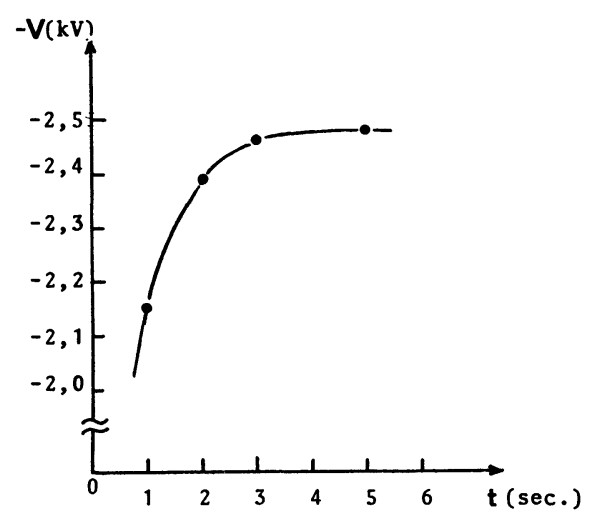

Fig. 3. - Potentiel de surface acquis en fonction de la durée du contact.

[Acquired surface potential vs. contact duration.]

chargé par contact pendant une durée $t$ avec une électrode portée à $-2,5 \mathrm{kV}$.

La surface ne se charge pas instantanément au contact, et quelques secondes sont nécessaires pour que le potentiel de surface après séparation atteigne sa valeur maximale, de l'ordre de $-2,5 \mathrm{kV}$.

Par ailleurs, on note (Fig. 4) une différence notable entre le comportement d'un échantillon métallisé et celui d'un échantillon non métallisé.

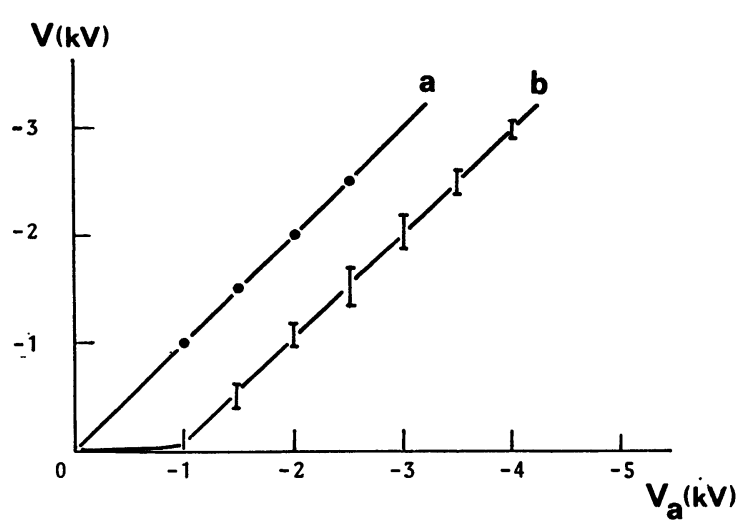

Fig. 4. - Potentiel mesuré après ouverture, en fonction du potentiel appliqué au contact : (a) échantillon métallisé ; (b) échantillon non métallisé.

[Measured potential after contact opening vs. applied potential : (a) metal coated sample; (b) non coated sample.]

Alors que l'échantillon métallisé acquiert rapidement, et conserve à quelques pourcents près à l'instant de la mesure, le potentiel $V$ de l'électrode HT, l'échantillon non métallisé présente à l'instant de la mesure un potentiel de l'ordre de $(V-1000)$ volts. En d'autres termes, l'échantillon non métallisé n'est pas chargé à saturation pendant le bref contact (on comprendra que sa surface n'a 
pas acquis assez de charges « libres »), et se décharge rapidement, conformément à la théorie de la section 2.1, avant de présenter un processus de décharge lente semblable à la décharge d'un échantillon métallisé.

3.2 DÉcharge NATURElle. - Une fois chargée, la surface se décharge beaucoup plus lentement que prévu par la relation (3). Ce déclin naturel lent du potentiel d'une couche isolante chargée a fait l'objet de nombreuses modélisations $[4,5]$, dont la plus simple est liée à la pénétration dans le matériau, sous l'action du champ entre la charge déposée et la charge d'influence sur la contre-électrode, d'une partie des charges libres déposées en surface. La figure 5 représente le cas peu réaliste mais simple où la charge déposée $q$, ici négative, pénétrerait sous forme d'un plan de charge sous l'action de son propre champ, dans l'hypothèse où le potentiel superficiel est mesuré par une sonde asservie, de sorte que le champ extérieur reste nul.

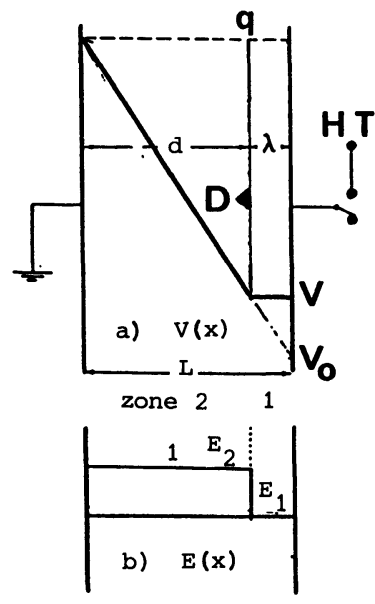

Fig. 5. - Modèle du plan de charge. Déclin du potentiel.

[The charge plane model. Potential decay.]

Dans le cadre restreint de ces hypothèses, le potentiel initial est $V_{0}=L q / \varepsilon$, et le plan de charge pénètre dans le matériau (c'est-à-dire vers la gauche sur la Fig.) sous l'action du champ constant $(-q / \varepsilon)$ à la vitesse $v=\mu|q| / \varepsilon, \mu$ étant la mobilité des charges. Ainsi, le potentiel décroît linéairement en valeur absolue selon la loi :

$$
V(t)=(L-\mu|q| t / \varepsilon) q / \varepsilon
$$

jusqu'à ce que le plan de charge atteigne la contreélectrode, à $t=L \varepsilon / \mu|q|$. Dans l'annexe 1, ceci sera validé et étendu au cas d'une charge répartie sur une profondeur $\lambda=L-d$.

Bien que très sommaire, cette analyse électrostatique du déclin du potentiel présente l'avantage considérable d'expliquer simplement le phénomène de « cross-over » observé lorsqu'on charge un même échantillon à des niveaux de potentiel différents. En effet, plus la tension initiale est élevée, plus le champ $q / \varepsilon$ est élevé, et plus le temps de transfert $L \varepsilon / \mu q$ est court, de sorte que les caractéristiques $V(t)$ se croisent. Toutefois, pour une valeur donnée du potentiel initial, la tension décroît d'autant plus rapidement que la charge est plus rapide. Ceci est probablement lié à l'élargissement du plan de charge, l'injection dans le matériau se saturant quand les charges déjà présentes sous la surface abaissent suffisamment le champ d'injection.

La figure 6 représente l'évolution du potentiel superficiel après ouverture du contact, sur un échantillon de PET (Mylar) de $36 \mu \mathrm{m}$ d'épaisseur, et sur un échantillon de polyéthylène de $50 \mu \mathrm{m}$.

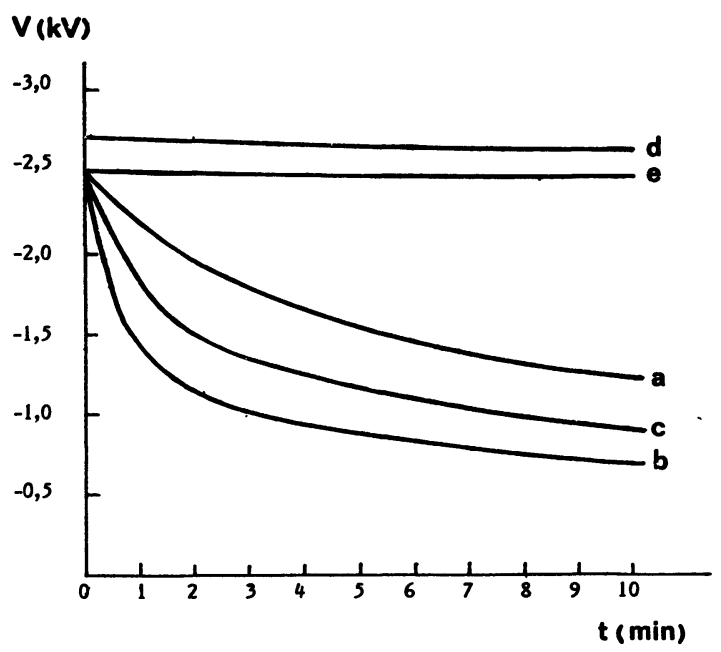

Fig. 6. - Evolution du potentiel après ouverture du contact: (a) polyéthylène non métallisé chargé $60 \mathrm{~s}$ à $-3500 \mathrm{~V}$; (b) PE métallisé chargé $1 \mathrm{~s}$ à $-2500 \mathrm{~V}$; (c) PE métallisé chargé $60 \mathrm{~s}$ à $-2500 \mathrm{~V}$; (d) polyester (PET) non métallisé chargé $60 \mathrm{~s}$ à $-3500 \mathrm{~V}$; (e) PET métallisé chargé $60 \mathrm{~s}$ à $-2500 \mathrm{~V}$.

[Surface potential behaviour after contact opening: (a) uncoated polyethylené charged $60 \mathrm{~s}$ at $-3500 \mathrm{~V}$; (b) coated PE charged $1 \mathrm{~s}$ at $-2500 \mathrm{~V}$; (c) coated PE charged $60 \mathrm{~s}$ at $-2500 \mathrm{~V}$; (d) uncoated PET charged $60 \mathrm{~s}$ at $2500 \mathrm{~V}$; (e) coated PET charged $60 \mathrm{~s}$ at $-2500 \mathrm{~V}$.

3.3 RETOUR DU POTENTIEL. - Si un échantillon est chargé pendant assez longtemps, puis court-circuité temporairement, on observe en général après l'ouverture du court-circuit une lente remontée du potentiel de la surface. Ceci est illustré par la figure 7. Il s'agit, en fait, d'un phénomène connu depuis très longtemps pour les chocs électriques que peut occasionner un condensateur HT après un court-circuit temporaire. Le phénomène, appelé « return potential» ou « residual voltage » par les 


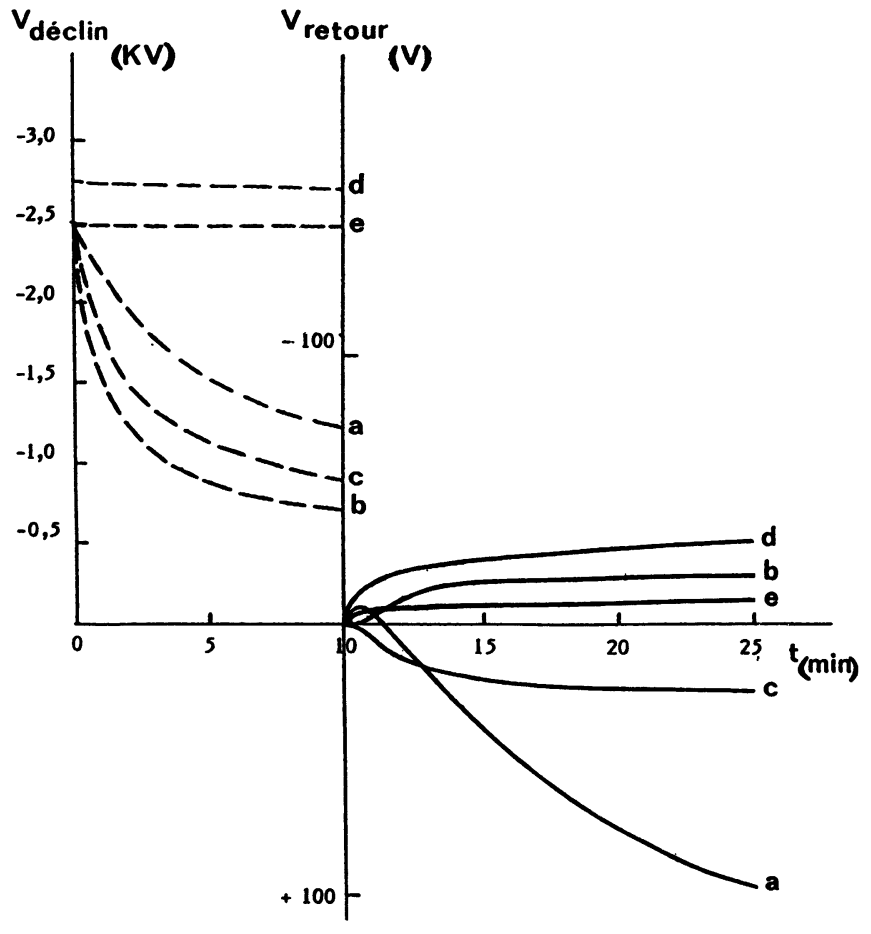

Fig. 7. - Retour du potentiel après court-circuit temporaire : (a) PE non métallisé (anomalies) ; (b) PE métallisé chargé $60 \mathrm{~s}$ à $-2500 \mathrm{~V}$; (c) PE métallisé chargé $60 \mathrm{~s}$ à $-2500 \mathrm{~V}$ (anomalie); (d) PET non métallisé chargé $60 \mathrm{~s}$ à $-2500 \mathrm{~V}$; (e) PET métallisé, chargé $60 \mathrm{~s}$ à $-2500 \mathrm{~V}$.

[Return potential after temporary short-circuit : (a) uncoated PE (anomalies); (b) coated PE charged $60 \mathrm{~s}$ at $-2500 \mathrm{~V}$; (c) coated PE charged $60 \mathrm{~s}$ at $-2500 \mathrm{~V}$; (d) uncoated PET charged $60 \mathrm{~s}$ at $-2500 \mathrm{~V}$; (e) coated PET charged $60 \mathrm{~s}$ at $-2500 \mathrm{~V}$.]

anglo-saxons, peut être interprété simplement dans le cadre du modèle électrostatique par le retour vers la surface de charges qui ont pénétré au-dessous pendant la période de déclin. En effet, comme le montre la figure 8 relative au modèle du plan de charge, le champ agissant sur ce plan passe de $E=-q / \varepsilon$ avant court-circuit à $E^{\prime}=E_{1}^{\prime}+E_{2}^{\prime}$, soit

$$
\begin{aligned}
E^{\prime}=d q / \varepsilon L+(d / L-1) & q / \varepsilon= \\
& =(2 d / L-1) q / \varepsilon
\end{aligned}
$$

après. Si $d / L>1 / 2$ (plan de charge initialement à droite du plan médian), le champ change donc de signe au court-circuit ; le plan de charge revient alors vers la surface à la vitesse constante $\boldsymbol{v}^{\prime}=$ $|\mu(2 d / L-1) q / \varepsilon|$, et le potentiel mesuré remonte linéairement vers la limite $V_{\infty}=L q / \varepsilon-V(0)$. On note en passant la relation simple existant entre la charge $q$ et la somme des potentiels: $q=$ $\left[V(0)+V_{\infty}\right] \varepsilon / L$.

L'annexe 2 étend le calcul et le résultat ci-dessus à une charge distribuée, et montre en outre comment la vitesse initiale de remontée du potentiel permet d'estimer la mobilité effective des charges. Des

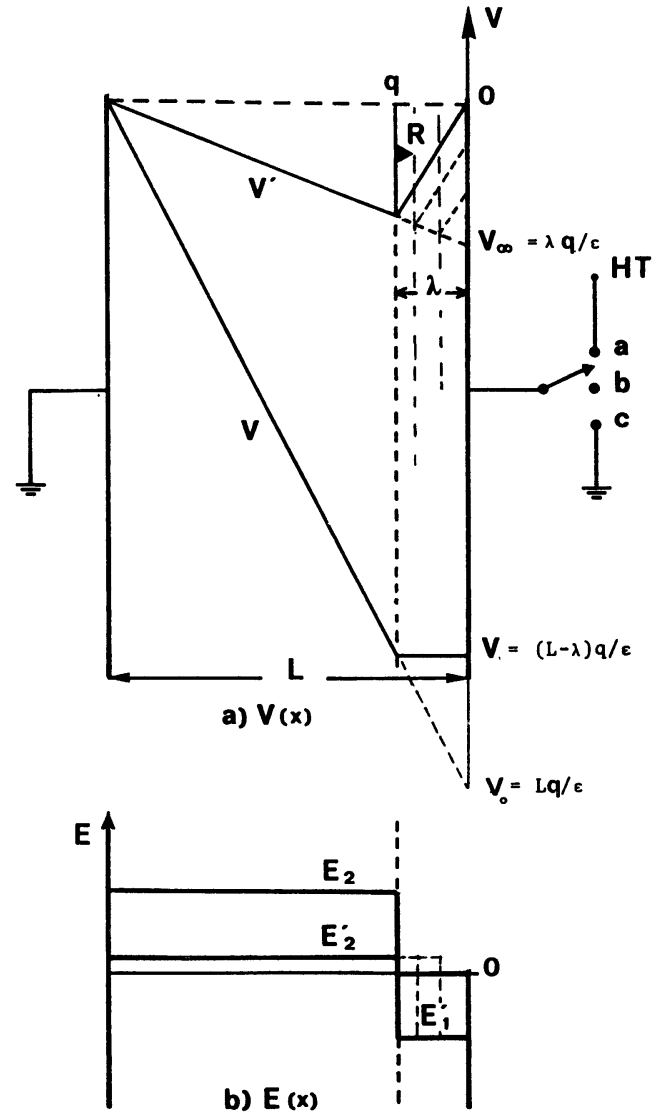

Fig. 8. - Modèle du plan de charge. Retour du potentiel après court-circuit temporaire, en supposant que seule $q$ est mobile. (a) Potentiel avant court-circuit $(V)$ et après $\left(V^{\prime}\right)$. (b) Champ avant CC $(E)$ et après $\left(E^{\prime}\right)$. Positions successives de l'interrupteur: $\mathrm{a} \rightarrow \mathrm{c} \rightarrow \mathrm{b}$.

[Charge plane model. Potential return after temporary short-circuit, assuming only $q$ mobile. (a) potential before short-circuit $(V)$ and after $\left(V^{\prime}\right)$; (b) field before s.c. $(E)$ and after $\left(E^{\prime}\right)$. Successive switch positions : $\mathrm{a} \rightarrow \mathrm{c} \rightarrow \mathrm{b}$.]

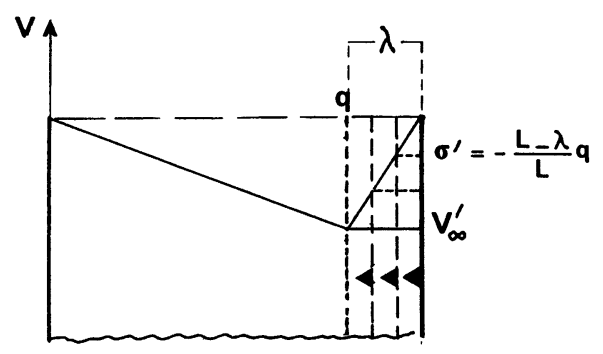

Fig. 9. - Idem figure 8 , mais dans l'hypothèse où seule la charge $\sigma^{\prime}$ de la surface libre est mobile ( $V$ seulement).

[As in figure 8, but assuming only charge on free surface $\left(\sigma^{\prime}\right)$ mobile.]

évolutions «anormales » du potentiel de retour, observables par exemple sur la figure 9, seront interprétées à la section 4 . 
3.4 ClAQUAGES DANS LA COUCHE D'AIR À LA SÉPARATION. - Enfin, on a montré à la section 2 que s'il n'existe pas de charges libres à l'interface, le potentiel de cette interface décroît très rapidement, causant le claquage de la lame d'air.

Or, les mesures de potentiel n'ont décelé aucune discontinuité au moment de la séparation, donc aucun claquage dans la lame d'air.

Il s'agit là d'une preuve supplémentaire de la présence de charges libres sur la surface après la charge par contact.

\section{Prise en compte d'autres mouvements de charges.}

Jusqu'ici, nous avons admis implicitement que seules les charges $q$ sont mobiles, et peuvent ainsi pénétrer dans l'échantillon pendant le déclin du potentiel, ou retourner vers la surface libre après court-circuit. Toutefois certaines observations telles que l'inversion du signe du retour de potentiel (Fig. 7, courbes a) et d)) montrent que d'autres plans de charges peuvent également migrer à travers l'échantillon. En fait, l'électrode flottante, non chargée avant le court-circuit, acquiert la charge $\sigma^{\prime}=-\frac{L-\lambda}{L} q$ au moment du court-circuit. D'autre part, la face à la masse, en influence totale avec $q$ avant le courtcircuit, porte alors une charge $(-q)$, qui devient $\sigma^{\prime \prime}=-\frac{\lambda}{L} q$ après le court-circuit. Si elles sont mobiles, ces charges contribuent à l'évolution du potentiel.

4.1 MOUVEMENT DU PLAN DE CHARGE $(-q)$ PENDANT LE DÉCLIN. - La situation avant le courtcircuit est décrite à la figure 5. Le seules charges en présence sont le plan de charge $q$, et le plan de charge $(-q)$ sur la face à la masse. Admettant que seule cette charge $(-q)$ est mobile, elle traverse l'échantillon sous l'influence du plan de charge $q$, et le potentiel superficiel décroît linéairement. Le déclin observé résulte des migrations en sens inverse de $q$ et de $(-q)$, dont les effets s'ajoutent, et bien entendu de l'élargissement des plans de charges, dont l'effet sera traité en annexes 1 et 2 .

4.2 MOUVEMENTS DES CHARGES $\sigma^{\prime}$ ET $\sigma^{\prime \prime}$ APRÈS LE COURT-CIRCUIT. - Nous avons vu qu'après le courtcircuit, les charges en présence sont :

$q$ à la profondeur $\lambda$

$\sigma^{\prime}=-(\bar{x} / L) q=-q(L-\lambda) / L$ sur la face libre $\sigma^{\prime \prime}=-(\lambda / L) q$ sur la face à la masse, et on vérifie que la charge $q$ est totalement écrantée.

Le mouvement de rappel de $q$ vers l'électrode libre a été étudié à la section 3.

Le mouvement de $\sigma^{\prime}$ vers l'intérieur transforme le profil du potentiel selon la figure 9 , et engendre une variation linéaire du potentiel de surface vers la valeur $\left({ }^{1}\right)$

$$
V_{\infty}^{\prime}=(\lambda / L) V=\lambda(L-\lambda) q / \varepsilon L
$$

dans le même sens que le rappel de la charge $q$ vers la face libre.

Par contre, le mouvement de $\sigma^{\prime \prime}$ vers l'intérieur de l'échantillon engendre une variation linéaire du potentiel en sens inverse (Fig. 10) vers la valeur

$$
V_{\infty}^{\prime}=-(\lambda / L) V=-\lambda(L-\lambda) q / \varepsilon L .
$$

On conçoit alors que si l'électrode du côté masse est plus injectante que la face libre, le potentiel de retour peut prendre le signe opposé à celui qui correspond au mouvement des charges $q$ et $\sigma^{\prime}$.

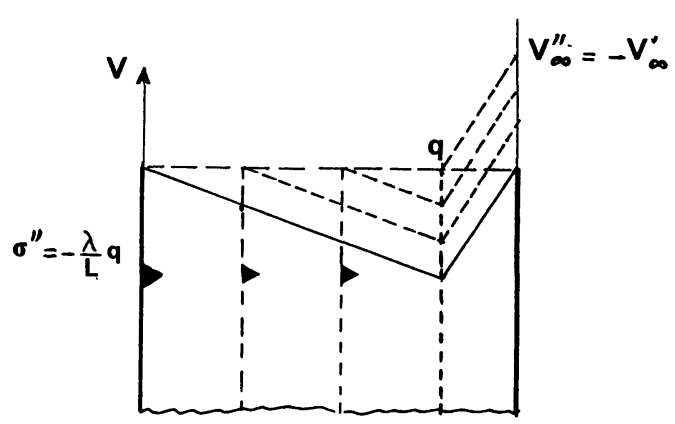

Fig. 10. - Idem, mais dans l'hypothèse où seule la charge $\sigma^{\prime \prime}$ de la face à la masse est mobile ( $V$ seulement).

[As above, but assuming only charge on grounded surface $\left(\sigma^{\prime \prime}\right)$ mobile.]

C'est probablement ce qui explique l'inversion observée (Fig. 9) du signe du potentiel de retour.

\section{Conclusion.}

Le potentiel qu'acquiert une surface isolante au contact d'une électrode métallique portée à haute tension résulte de l'accumulation sur l'isolant de charges libres injectées par le métal. Les processus d'accumulation et de dissipation de ces charges sont en fait semblables à ceux qui opèrent dans la charge par effet couronne [6].

Après l'ouverture du contrat, le potentiel acquis par la surface décroît beaucoup plus lentement que ne le prévoit le modèle simple de l'ouverture sans charges libres, et cette lente décroissance est due, comme dans le cas d'un échantillon chargé par effet couronne, à la pénétration à l'intérieur de l'échantillon des charges déposées à sa surface.

Pour un matériau donné, la vitesse relative du déclin $\mathrm{d} V / V \mathrm{~d} t$ est d'autant plus rapide que le niveau de charge est élevé, de sorte que des courbes

(1) Dans tout ce qui suit, $V(0)$ est noté $V$. 
de $V(t)$ correspondant à des niveaux de charge différents se croisent. Ce phénomène de «crossover », particulièrement net avec le polyéthylène, s'explique très simplement par le modèle du plan de charge migrant à travers l'échantillon sous l'action de la force-image sur la contre-électrode. Ce modèle simpliste est perfectionné dans les Annexes pour tenir compte de l'épaisseur de la charge.

Les mesures du potentiel de surface juste avant et longtemps après un court-circuit temporaire de l'échantillon permettent de caractériser les charges injectées. Plus précisément, la quantité globale des charges injectées dans l'échantillon et la profondeur moyenne de ces charges peuvent être déduites des mesures de potentiel à l'aide de relations très simples. Le bien-fondé de cette technique a été démontré dans la publication antérieure [6] citée plus haut, avec des échantillons chargés à une profondeur connue par des faisceaux d'électrons monocinétiques.

Enfin, la mobilité effective des charges, déduite des vitesses d'évolution du potentiel, est en bon accord avec les résultats obtenus par d'autres méthodes, généralement plus complexes.

Quelques différences mineures mais significatives entre les processus d'injection de charge par un contact métallique et un plasma d'effet couronne seront discutées dans une prochaine publication.

\section{Annexe 1.}

DÉCLIN DU POTENTIEL POUR UNE CHARGE DISTRIBUÉE. - Sur la figure 11, une densité de charge $\rho(x)$ est distribuée initialement entre $x=d$ et $x=L$ :

$$
q=\int_{d}^{L} \rho(x) \mathrm{d} x
$$

$E(x)$ désignant le champ dans l'intervalle $(x, d)$ et $E$ le champ uniforme pour $0 \leqslant x \leqslant d$, la continuité du potentiel s'écrit :

$$
E d+\int_{d}^{L} E(x) \mathrm{d} x=-V
$$

Une intégration par parties tenant compte de la relation de Poisson et de la condition limite $E(L)=0$ transforme (A.2) en :

$$
\frac{1}{\varepsilon} \int_{d}^{L} x \rho(x) \mathrm{d} x=V
$$

En appelant $\bar{x}=\frac{1}{q} \int_{d}^{L} x \rho(x) \mathrm{d} x$ l'abscisse du centroïde de la charge, (A.3) devient :

$$
V=\bar{x} \frac{q}{\varepsilon} .
$$
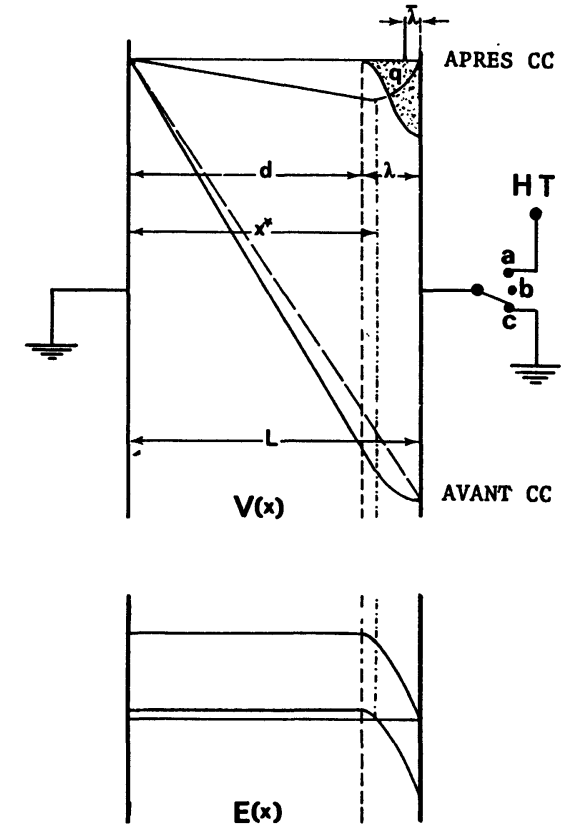

Fig. 11. - Modèle de la charge répartie (cf. annexes 1 et 2).

[Distributed charge model (cf. appendices 1 and 2).]

Le potentiel de surface ne décroît plus linéairement comme dans le cas du plan de charge. Toutefois, à l'instant initial, le taux de décroissance peut se calculer, par extension du texte, au moyen de la relation :

$$
(\mathrm{d} V / \mathrm{d} t)_{0}=\frac{\mu}{\varepsilon} \int_{d}^{L} \rho(x) E(x) \mathrm{d} x
$$

avec

$$
E(x)=-q(x) / \varepsilon=\left[q^{\prime}(x)-q\right] / \varepsilon
$$

et

$$
q^{\prime}(x)=\int_{d}^{x} \rho(x) \mathrm{d} x .
$$

La relation (A.5) peut alors s'écrire :

$$
(\mathrm{d} V / \mathrm{d} t)_{0}=\frac{\mu}{\varepsilon^{2}}\left[\int_{d}^{L} \rho(x) q^{\prime}(x) \mathrm{d} x-q^{2}\right]
$$

c'est-à-dire :

$(\mathrm{d} V / \mathrm{d} t)_{0}=\frac{\mu}{\varepsilon^{2}}\left[\int_{x=d}^{x=L} q^{\prime} \mathrm{d} q^{\prime}-q^{2}\right]=-\mu q^{2} / 2 \varepsilon^{2}$

ou encore, en utilisant (A.4) :

$$
(\mathrm{d} V / \mathrm{d} t)_{0}=-\frac{\mu}{2}(V / \bar{x})^{2}
$$

en notant que $\mu$ a le signe des charges. 
Notons que si $\bar{x} / L \sim 1$ (charge peu profonde),

$$
(\mathrm{d} V / \mathrm{d} t)_{0} \sim-\mu V^{2} / 2 L^{2} \text {. }
$$

\section{Annexe 2.}

RETOUR DU POTENTIEL APRÈS COURT-CIRCUIT. Pendant le bref court-circuit, le potentiel à $x=L$ est ramené à 0 sans que la distribution $\rho(x)$ des charges ne soit affectée. Le champ $E(x)$ dans l'échantillon devient alors :

$$
E^{\prime}(x)=E(x)+V / L
$$

et, après le court-circuit, le potentiel remonte de 0 à la valeur limite $V_{\infty}$ donnée par:

$$
V_{\infty}=L q / \varepsilon-V .
$$

On retombe sur la même relation que pour le plan de charge, excepté qu'ici le potentiel ne remonte pas linéairement.

En combinant (A.4) et (A.5), on obtient :

$$
V_{\infty}=\left(\frac{L}{\bar{x}}-1\right) V
$$

relation qui permet en principe de calculer la profondeur initiale de la charge connaissant $V, V_{\infty}$ et $L . \mathrm{Si}$ $V_{\infty} / V$ est très petit par rapport à l'unité, $x \sim L$ et la charge est peu profonde.

Par ailleurs, la vitesse initiale de remontée du potentiel est comme plus haut mais avec la nouvelle valeur du champ :

$(\mathrm{d} V / \mathrm{d} t)_{0}=\frac{\mu}{\varepsilon} \int_{d}^{L} \rho(x)[V / L-q(x) / \varepsilon] \mathrm{d} x$.

En utilisant les résultats obtenus dans l'annexe 1, la relation (A.12) devient :

$$
(\mathrm{d} V / \mathrm{d} t)_{0}^{\prime}=(\mathrm{d} V / \mathrm{d} t)_{0}+\mu V^{2} / L \bar{x}
$$

ou encore

$$
(\mathrm{d} V / \mathrm{d} t)_{0}^{\prime}=\left(\frac{\bar{x}}{L}-\frac{1}{2}\right) \frac{\mu V^{2}}{\bar{x}^{2}} .
$$

On retrouve le fait que le potentiel remonte, en valeur absolue, si le centroïde de la charge initiale est situé à droite du plan médian. Si $\bar{x} / L \sim 1$, $(\mathrm{d} V / \mathrm{d} t)_{0}^{\prime} \sim \mu V^{2} / 2 L^{2}$. On retrouve dans ce cas la même valeur approchée, mais de signe opposé, que pour le déclin.

\section{Bibliographie}

[1] LoEB, L. B., Static Electrification (Springer Verlag, Berlin) 1958

[2] HARPER, W. R., Contact and frictional electrification (Clarendon Press, Oxford) 1967.

[3] Rose-InNes, A. and Lowell, J., Contact electrification, Adv. Phys. 29 (1980) 947.

[4] Davies, D. K., The generation and dissipation of static charges, Static Electrification Conference (1967) p. 29.
[5] Coelho, R., Levy, L. and Sarrail, D., On the natural decay of corona charged insulating sheets, Phys. Status Solidi 94 (1986) 289.

[6] Coelho, R., Jestin, P., Levy, L. and Sarrail, D., On the return voltage buildup in insulating materials, Trans. IEEE Insulation, 22 (1987) 683. 